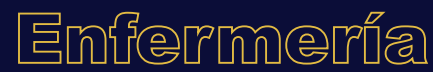

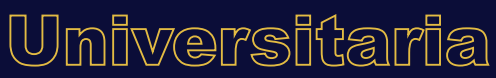

\section{Diseño y validación de material didáctico para una intervención educativa de enfermería en cuidadores primarios}

\author{
Design and validation of didactic \\ materials for a nursing educational \\ intervention to primary caregivers
}

\section{Desenho e validação de material didático para uma intervenção educativa de enfermagem em cuidadores primários}

\section{E. Velazquez-Moreno ${ }^{a *}$, M.S. González-Velázquez ${ }^{b}$, B. De la Peña-León ${ }^{c}$}

ORCID

${ }^{\mathrm{a}} \underline{0000-0003-4630-4758}$
${ }^{\mathrm{b}} \underline{0000-0002-5851-4886}$
${ }^{\mathrm{c}} \underline{0000-0002-2579-7699}$

Universidad Nacional Autónoma de México, Facultad de Estudios Superiores Zaragoza, Carrera de Enfermería, Ciudad de México, México

Recibido: 10 noviembre 2019

Aceptado: 28 septiembre 2020

RESUMEN

Introducción: El cuidador es una persona que en sí misma requiere cuidado, existe poca evidencia sobre la validación de material dirigido a cuidadores primarios de adultos en el domicilio.

Objetivo: Diseñar y validar el material didáctico de apoyo en una propuesta de intervención educativa de enfermería para mejorar el autocuidado de cuidadores primarios de personas con enfermedades crónicas no transmisibles.

*Autor para correspondencia. Correo electrónico: eduardovemo3@gmail.com https://doi.org/10.22201/eneo.23958421e.2020.4.787

1665-7063/@ 2020 Universidad Nacional Autónoma de México, Escuela Nacional de Enfermería y Obstetricia. Este es un artículo Open Access bajo la licencia CC BY-NC-ND (http://creativecommons.org/licenses/by-nc-nd/4.o/). 
Material y Métodos: Investigación metodológica para validación de diez carteles. Evaluación por cuatro expertos y diez cuidadores primarios. La investigación se desarrolló en cuatro fases: a) valoración de las necesidades en salud y determinación de los contenidos, b) elaboración de material didáctico, c) jueceo y validación técnica, d) validación con población. Se evaluaron los aspectos de atracción, comprensión, involucramiento y aceptación con un cuestionario dicotómico de ocho preguntas por cartel. Se calculó el coeficiente $\mathrm{V}$ de Aiken como indicador de aprobación. Se tomó el valor de 0.7 y superior como punto para la validación.

Resultados: En la primera ronda de evaluación por los expertos se modificaron tres carteles por su bajo coeficiente de comprensión-atracción y se reelaboró un cartel. En la segunda versión de los carteles se alcanzaron coeficientes superiores a 0.81. Sin embargo, los cuidadores puntuaron dos carteles con valores inferiores al estándar, por lo que luego de tomar su opinión al respecto se modificaron aspectos de diseño.

Discusión: Las recomendaciones de los expertos en el área y particularmente los intereses de los cuidadores primarios fueron la base para mejorar el diseño del material didáctico. Conclusiones: Tras ajustar elementos de la atracción y comprensión de los carteles se concluyó que el Material Didáctico (MD) es adecuado para su implementación en una Intervención Educativa (IE) dirigida a mejorar el autocuidado de cuidadores primarios.

Palabras clave: Cuidadores; educación en salud; estudio de validación; México.

\section{ABSTRACT}

Introduction: Caregivers are persons who themselves also need care, and there is few evidence regarding the validation of educational materials for caregivers of adult home care.

Objective: To design and validate supporting didactic materials for a nursing educational intervention proposal in order to improve self-care among primary caregivers attending patients with non-transmissible chronic illnesses.

Materials and Methods: This is a validating research study using 10 educational materials which were assessed by 4 field experts and 10 primary caregivers. The research study was developed in four stages: a) assessment of the health needs and determination of the contents for the education materials, b) elaboration of the education materials, c) technical validation, d) assessment on the use in a population. Features of attractiveness, comprehension, involvement, and acceptance were estimated using a dichotomous questionnaire of 8 questions for each educational material. The Aiken V coefficient of approval was calculated. Values greater than 0.7 were considered as valid results.

Results: In the first round of assessment by the experts, three educational materials were modified due to their low coefficient of comprehension-attractiveness and one education material was redesigned. The subsequent version of the education materials showed coefficients higher than 0.81 , however, caregivers gave two materials scores below the standard, and these materials were further redesigned.

Discussion: The recommendations from the experts in the field, along with the interests of the primary caregivers, were the base to improve the design of the didactic materials. 
Conclusions: After adjusting elements related to the attractiveness and comprehension of the didactic materials, it was concluded that the redesigned materials were adequate for an educational intervention aimed at improving self-care among primary caregivers.

Keywords: Caregivers; health education; validation study; Mexico.

\section{RESUMO}

Introdução: O cuidador é uma pessoa que em si mesmo requer de cuidado, há pouca evidência sobre a validação de material dirigido aos cuidadores primários de adultos no domicílio.

Objetivo: Desenhar e validar o material didático de apoio em uma proposta de intervenção educativa de enfermagem para melhorar o autocuidado de cuidadores primários de pessoas com doenças crônicas não transmissíveis.

Material e Métodos: Pesquisa metodológica para validação de dez pôsteres. Avaliação por quatro especialistas e dez cuidadores primários. A pesquisa desenvolveu-se em quatro fases: a) avaliação das necessidades de saúde e determinação dos conteúdos, b) elaboração do material didático, c) julgamento e validação técnica, d) validação com população. Avaliaram-se os aspectos de atração, compreensão, envolvimento e aceitação com um questionário dicotômico de oito perguntas por pôster. Foi calculado o coeficiente $\mathrm{V}$ de Aiken como indicador de aprovação. Tomou-se o valor de 0.7 e superior como ponto para a validação.

Resultados: Na primeira rodada de avaliação dos especialistas modificaram-se três pôsteres por seu baixo coeficiente de compreensão-atração e foi reelaborado um pôster. Na segunda versão dos pôsteres foram alcançados coeficientes superiores a 0.81 . Porém, os cuidadores pontuaram dois pôsteres com valores inferiores ao padrão, portanto, após se manifestarem sobre o assunto modificaram-se aspectos de desenho.

Discussão: As recomendações dos especialistas da área e particularmente, os interesses dos cuidadores primários foram a base para melhorar o desenho do material didático.

Conclusões: Após ajustar elementos da atração e compreensão dos pôsteres foi concluído que o MD é adequado para sua implementação em uma IE dirigida a melhorar o autocuidado de cuidadores primários.

Palavras chave: Cuidadores; educação em saúde; estudo de validação; México.

\section{INTRODUCCIÓN}

Los sistemas de salud en el mundo se enfrentan a una gran demanda de atención sanitaria, prueba de esto son las cifras reportadas en años recientes. Por ejemplo, en 2014 cerca de 422 millones de personas vivían con Diabetes Mellitus, un año más tarde se reportó que al menos 200 millones de personas ya habían superado su esperanza de vida saludable y que 110 millones de personas vivían con alguna discapacidad grave. Aunado a ello, las proyecciones en salud del año 2018 previeron un aumento considerable en la prevalencia de las condiciones anteriores ${ }^{1-3}$.

En este panorama desafiante, el cuidado en el domicilio es un elemento de gran trascendencia que repercute en la atención cotidiana de las personas en tales condiciones y que no debería quedar en segundo plano. A pesar de que la mayor parte del cuidado en el mundo se proporciona en el hogar, el cuidado domiciliario en ocasiones se vuelve invisible, ya que en su mayoría se lleva 
a cabo por mujeres, de manera informal y de forma no remunerada4. Es posible que la razón por la que se subestima sea la naturaleza misma del cuidado, pues al menos $80 \%$ de éste implica realizar labores domésticas y únicamente $20 \%$ restante es cuidado directo en sí, es decir, prestar ayuda en la enfermedad, la discapacidad o la dificultad para realizar actividades de la vida diaria 4,5.

Con frecuencia los miembros de una familia son los que se convierten en cuidadores, cuyo cambio puede ser gradual y casi imperceptible para el cuidador. En ocasiones se comienza a cuidar en etapas preclínicas o bien puede iniciar el cuidado súbitamente, en ambos casos el cuidado puede prolongarse por décadas5. Si bien la motivación para el cuidado del familiar generalmente es altruista, la relación previa entre el cuidador y la persona a quien se cuida es un aspecto importante que puede marcar una diferencia sustancial, dependiendo de si esta relación ha sido positiva o negativa4.

En México el cuidado en el domicilio es administrado por 286 mil cuidadores, en su mayoría del género femenino con una edad alrededor de los 40 años, quienes cuentan con estudios de secundaria o inferiores y poco más de la mitad de ellos no tienen pareja. El cuidado que proporcionan implica cerca de 40 horas semanales y en la mayoría de los casos perciben entre uno y dos salarios mínimos ${ }^{6}$.

Es así que las características personales de quien cuida, la naturaleza de la relación previa entre quien cuida y quien es cuidado, las necesidades de cuidado en el hogar (tanto directo como indirecto) y el tiempo que éste demanda, generan un escenario complicado para llevar a cabo el cuidado, particularmente en situaciones en las que éste debe continuarse por un periodo prolongado como en las Enfermedades Crónicas No Transmisibles (ECNT), en el cuidado en la vejez y la discapacidad grave4-6.

Es frecuente que, en estas condiciones, el cuidador primario experimente diversos problemas de salud como estrés, agotamiento, nerviosismo, insomnio, entre otros que en conjunto han sido descritos como cansancio del rol del cuidador?. Diversos trabajos realizados por profesionales de la salud pretenden detectar, disminuir y prevenir estos problemas de salud, una de las metodologías más comunes para lograr tal fin es la investigación con intervención.

Entre las intervenciones dirigidas al cuidador primario, la intervención educativa (IE) es comúnmente elegida por su bajo costo, relativa facilidad de implementación y versatilidad ${ }^{8-10}$. Sin embargo, es común encontrar en la literatura científica que buena parte de las IE se encamina a la mejoría de las habilidades del cuidador para cuidar ${ }^{9,11-13}$, seguido de IE dirigidas a mejorar el conocimiento sobre la enfermedad, condición o padecimiento de las personas a quien cuidan ${ }^{14-16}$. Es decir, el cuidador es considerado parte del sistema de apoyo de la persona con necesidad de cuidado y no como una persona que en sí misma requiere cuidado ${ }^{17}$.

Por otra parte, en publicaciones sobre IE en enfermería se detalla el desarrollo e implementación de material didáctico $(M D)^{18-21}$, pero existe poca evidencia sobre la validación de material dirigido a cuidadores primarios de adultos en el domicilio. Finalmente, algunas IE presentan como objetivo mejorar la salud del cuidador en diversos aspectos como: la disminución del cansancio, el insomnio, el estrés, el desgaste físico, emocional o en la sobrecarga del cuidado ${ }^{22,23}$. Con estos objetivos se busca mejorar un aspecto trascendental en la vida de los cuidadores; sin embargo, en pocas IE se puede apreciar una intención preventiva o que permita un estado de salud sostenido, lo cual es de particular interés para fortalecer al cuidador primarioº ${ }^{24}$

De acuerdo a diversas guías sobre diseño, desarrollo y validación de $\mathrm{MD}$, se debe tener una excelente valoración sobre las necesidades en salud de los cuidadores a quienes se dirige el MD, 
realizar un consenso entre personal de salud y cuidadores sobre los contenidos que se abordarán, privilegiando las preferencias de los cuidadores y aspectos culturales, contar con criterios de validación técnica en el desarrollo de MD, así como de un proceso de evaluación continua respecto a su efectividad ${ }^{25-28}$.

En el caso del presente trabajo, el MD dirigido a cuidadores primarios en el domicilio fue diseñado para ser el apoyo de una propuesta de intervención educativa de enfermería en la que se contemplaron: a) los requisitos universales de autocuidado, entendidos como "el grado de cumplimiento de los cuidados de salud y capacidad el individuo para llevarlos a cabo", b) el desarrollo de la agencia de autocuidado entendida como "la capacidad que permite a las personas discernir los factores que deben ser controlados o tratados para regular su propio funcionamiento y desarrollo, de manera que cubran sus requisitos de autocuidado" y c) la autoeficacia percibida ${ }^{29,30}$. Esta última representa la percepción de competencia de uno mismo para ejecutar una cierta conducta e influye sobre las barreras de acción percibidas, de manera que la mayor eficacia lleva a percibir menores barreras para el desempeño de esta conducta ${ }^{31}$.

Conforme a lo anterior, se consideraron las siguientes premisas planteadas en la teoría del déficit del autocuidado de Elizabeth Dorothea Orem y el Modelo de promoción de la salud de Nola J Pender:

a) la autoeficacia percibida es un buen indicador en el logro del objetivo de intervenciones educativas dirigidas al autocuidado ${ }^{31}$.

b) el grado de desarrollo de la agencia de autocuidado, del cumplimiento óptimo de los requisitos universales de autocuidado de los cuidadores son claves para el logro y mantenimiento del autocuidado 29,30 . A raíz de lo que se consideró plausible:

c) el grado de desarrollo de la autoeficacia percibida es clave en el mantenimiento del autocuidado de los cuidadores primarios.

\section{MATERIAL Y MÉTODO}

Diseño: Investigación metodológica cuyo objetivo fue el diseño y validación del MD de apoyo en una propuesta de IE de enfermería para mejorar el autocuidado de 26 cuidadores primarios de personas con ECNT de dos comunidades al oriente de la Ciudad de México. Se consideró la metodología planteada por Martínez, Palmar y Pedraz en 2014 para el desarrollo de un proyecto de educación para la salud y la elaboración de sesiones educativas ${ }^{32}$. Para la elaboración del material didáctico se llevaron a cabo cuatro fases que se describen a continuación.

Fase 1: valoración de las necesidades en salud y determinación de los contenidos.

Se desarrolló y aplicó un cuestionario dirigido a los cuidadores, con 36 ítems sobre autocuidado y autoeficacia del cuidador, compuesto por tres dimensiones (la agencia de autocuidado, de los requisitos universales y de la autoeficacia percibida) el cual presentó un índice de consistencia interna de 0.854 a través de la prueba estadística de Alfa de Cronbach. La determinación del desarrollo de las tres dimensiones se escaló en tres niveles: elemento poco desarrollado, elemento en desarrollo y elemento desarrollado. Las características de los cuidadores se describen a continuación:

- La agencia de autocuidado se encuentra en desarrollo en la mayoría de los cuidadores (58\%), particularmente en los aspectos relacionados a la administración de energía en el día a día, la búsqueda de información relacionada con el cuidado y las habilidades que fortalecen el autocuidado. 
- Los requisitos universales de autocuidado se encuentran en desarrollo en la mayoría de los cuidadores (65\%), principalmente en la promoción del funcionamiento y el desarrollo humano dentro de los grupos sociales de acuerdo con el potencial humano, el conocimiento de las limitaciones y el deseo humano de ser normal.

- La autoeficacia percibida de los cuidadores primarios se encuentra desarrollada en la mayoría de los participantes (54\%) y en desarrollo en los demás (46\%).

La elección de la temática a abordar en la IE de la que derivó el material didáctico se basó en las características descritas, en este caso sus necesidades en salud, tal como lo plasman Martínez, Palmar y Pedraz ${ }^{32}$. Se optó por cinco sesiones educativas con duración planeada de 20 minutos con dos carteles cada una. Los contenidos fueron los siguientes: El autocuidado: una práctica para toda la vida (carteles 1 y 2); Fuentes de información para el autocuidado (carteles 3 y 4); Habilidades que fortalecen el autocuidado (carteles 5 y 6); Equilibrio del tiempo, pasatiempos, bienestar y cuidado (carteles 7 y 8); La razón y beneficios del autocuidado (carteles 9 y 10).

\section{Fase 2: elaboración de material didáctico.}

Con base en los criterios de elaboración de material didáctico propuestos por Guerra y Alva para la $\mathrm{OPS}^{26}$, se crearon diez carteles como apoyo para la IE. Para su elaboración se buscó que los aspectos de tamaño, color, ilustraciones y tipo de letra se adecuaran a las características de los cuidadores. Para el diseño de las ilustraciones del MD se realizó una lista de personajes, situaciones y elementos visuales que se entregaron a un diseñador gráfico para su elaboración. Los carteles se crearon con el programa Microsoft Publisher 2013 en medidas de $120 \mathrm{~cm}$ verticales y $90 \mathrm{~cm}$ horizontales.

\section{Fase 3: jueceo y validación técnica.}

Se realizaron pruebas de validación técnica con la participación de cuatro expertos provenientes de la Escuela Nacional de Enfermería y Obstetricia y las Facultades de Estudios Superiores Iztacala y Zaragoza. Los expertos contaban con estudios de doctorado y experiencia en las áreas de: educación para la salud, educación en enfermería y/o el desarrollo de intervenciones educativas.

Se hizo entrega de un cuadernillo engargolado a cada experto que contenía una explicación breve sobre la temática, problematización y objetivos de una intervención educativa de enfermería y la primera versión de los diez carteles que componen el MD en escala de 1 a 4.21 (1:4.21), así como instrucciones para que ellos emitieran su juicio sobre el cumplimiento de los carteles en torno a los siguientes cuatro criterios:

- Atracción: consiste en averiguar si el material llama la atención del público al cual está dirigido. Esto es, si le agradan los colores, el diseño y formato. Se evaluaron dos preguntas a los expertos: 1. ¿El cartel llamará la atención del cuidador?, 2. ¿El diseño, formato y colores son adecuados para los contenidos?

- Comprensión: permite conocer si el público entiende los contenidos y mensajes presentados y si le queda claro el mensaje que se le propone. Se evaluaron dos preguntas a los expertos: 3. ¿Se entienden los contenidos y mensajes presentados en el cartel?, 4. ¿Considera que al leer los mensajes del cartel éstos le quedarán claros al cuidador?

- Involucramiento: este criterio busca averiguar si el público objetivo siente que el material que se valida es para personas como ellos, es decir, si se siente identificado con lo que le proponen los mensajes. Se evaluaron dos preguntas a los expertos: 5. ¿Considera que los 
cuidadores se sentirán identificados con el mensaje del cartel?, 6. ¿Considera que los mensajes del cartel aplican al contexto del cuidador?

- Aceptación: este componente permite confirmar que nada moleste o genere alguna indisposición. Indica si acepta plenamente el enfoque, los contenidos y lenguaje utilizado. Se evaluaron dos preguntas a los expertos: 7. ¿Considera que las recomendaciones dadas en el cartel son adecuadas para el cuidador?, 8. ¿Piensa que el cuidador aceptará el mensaje y los contenidos?

Tras obtener las respuestas a los criterios de evaluación del MD se calculó el coeficiente V de Aiken como prueba de validez de contenido, éste índice tiene como puntuación máxima 1 y como mínima 0. Para su aceptación en esta investigación se determinó como punto de corte un índice de 0.7 o superior por criterio, es decir, que para que uno de los criterios fuera válido debía obtenerse un grado de acuerdo entre observadores de por lo menos 70\%. Lo anterior corresponde a la metodología seguida por Correa Tello en 2014, quien da cuenta de su uso común en otras investigaciones33.

Teniendo en cuenta las observaciones de los expertos y los coeficientes más bajos, se realizaron ajustes al contenido, distribución de las imágenes y colores en el MD, con la finalidad de mejorar su calidad y alcanzar el índice mínimo establecido de 0.7 .

\section{Fase 4: validación con población.}

Para comprobar si el contenido y la forma del MD funcionan, se realizó una encuesta individual por medio de una plataforma digital a un grupo representativo del público objetivo, compuesto por diez cuidadores primarios de personas con ECNT. Las características de los cuidadores se describen en la tabla 1.

Por medio de la plataforma se les presentaron los diez carteles del MD en formato digital, seguido de ocho preguntas sobre los criterios de atracción, comprensión, involucramiento y aceptación. Tras obtener las respuestas se calculó el coeficiente V de Aiken para determinar el grado de acuerdo entre observadores.

Con base en las observaciones de los cuidadores y en los dos coeficientes más bajos, se realizaron ajustes a los dos carteles con la finalidad de mejorar su calidad y alcanzar el índice mínimo establecido de 0.7 .

\section{Consideraciones éticas:}

De acuerdo con el artículo 117 del Reglamento de la Ley General de Salud en Materia de Investigación³4, se consideró la presente investigación como una investigación sin riesgo, se siguió lo estipulado en los artículos 13, 14, 16 y 116 del reglamento mencionado y se guardaron los principios bioéticos de justicia, beneficencia, no maleficencia y autonomía descritos por Beauchamp y Childrens ${ }^{35}$.

A su vez, se realizó la invitación a los expertos para participar en el proceso de validación por medio de una carta escrita y firmada por los autores de la presente investigación. Se explicó a los participantes el alcance de la investigación, la naturaleza voluntaria de su participación, así como el nulo riesgo que esto conllevó y tras aclarar dudas se obtuvo su consentimiento informado para la aplicación de las encuestas.

\section{RESULTADOS}

Características de los cuidadores: en la validación con población del MD participaron diez cuidadores de personas con ECNT, hubo predominio del género femenino, con ocupación principal 
de ama de casa y edades entre los 33 y 50 años con media de 41.3 y desviación estándar de 6.3 años. Respecto al tiempo que llevan como cuidadores varía desde 5 meses hasta 13 años (Tabla 1).

Validación de los expertos: en la tabla 2 se presenta el indice de aprobación de los carteles en su primera versión, los cuales mostraron en forma consistente valores cercanos a 1 en los criterios de involucramiento y aceptación. Por otra parte, los criterios de comprensión y atracción presentaron índices más bajos. Se muestran también los índices obtenidos por cartel, en los que el tercero de ellos presenta un valor por debajo de 0.7, además se observa el coeficiente por contenido, es decir, por tema a abordar en la IE. Las observaciones cualitativas de los carteles 1, 4 y 6 se presentan en la tabla 3.

Después de lo reportado por los expertos en las tablas 2 y 3 , se modificaron aspectos poco favorables del material didáctico de los carteles 1, 4 y 6, además se rediseñó el cartel 3, tras lo cual se obtuvieron los valores de la tabla 4, en la cual se observa una aprobación por cartel superior a o.8 en cada cartel y en cada contenido.
Tabla 1.

Características

de los cuidadores

\begin{tabular}{ll} 
Características & Fr (\%) \\
\hline Género & $8(80)$ \\
\hline Femenino & $2(20)$ \\
\hline Masculino & $7(70)$ \\
\hline Ocupación & $2(20)$ \\
\hline Empleado casa & $1(10)$ \\
\hline Profesionista & $5(50)$ \\
\hline Edad & $3(30)$ \\
\hline De 30 a 39 años & $2(20)$ \\
\hline De 40 a 49 años & \\
\hline 50 años o más & $3(30)$ \\
\hline Tiempo cuidando & $2(20)$ \\
\hline Menos de un año & $2(20)$ \\
\hline De 1 a 2 años & $3(30)$ \\
\hline De 2 a 5 años
\end{tabular}

Validación de los cuidadores: la aprobación de los cuidadores se aprecia en la tabla 5, en la que se observan índices iguales o superiores a 0.7 para los criterios de atracción, comprensión, involucramiento y aceptación en los carteles 2-9, lo que contrasta con los carteles 1 y 10. El cartel 1 resultó poco atractivo, con bajo nivel de involucramiento y aceptación, además, junto con el cartel 2 presentaron un índice de aprobación por contenido de 0.67. Ambos se diseñaron para la sesión

Tabla 2. Índice de aprobación por los jueces en la primera versión del material didáctico

\begin{tabular}{|c|c|c|c|c|c|}
\hline Contenidos & $\begin{array}{l}\text { 1. El autocuidado: } \\
\text { una práctica para } \\
\text { toda la vida }\end{array}$ & $\begin{array}{l}\text { 2. Fuentes de } \\
\text { información } \\
\text { para el } \\
\text { autocuidado }\end{array}$ & $\begin{array}{l}\text { 3. Habilidades } \\
\text { que fortalecen } \\
\text { el autocuidado }\end{array}$ & $\begin{array}{l}\text { 4. Equilibrio } \\
\text { del tiempo, } \\
\text { pasatiempos, } \\
\text { bienestar y } \\
\text { cuidado }\end{array}$ & $\begin{array}{l}\text { 5. La razón y } \\
\text { beneficios del } \\
\text { autocuidado }\end{array}$ \\
\hline
\end{tabular}

\begin{tabular}{lllllllllcc} 
Criterios/ & Cartel & Cartel & Cartel & Cartel & Cartel & Cartel & Cartel & Cartel & Cartel & Cartel \\
cartel* & $\mathbf{1}$ & $\mathbf{2}$ & $\mathbf{3}$ & $\mathbf{4}$ & $\mathbf{5}$ & $\mathbf{6}$ & $\mathbf{7}$ & $\mathbf{8}$ & $\mathbf{9}$ & $\mathbf{1 0}$ \\
\hline Atracción & 0.75 & 0.87 & 0.5 & 0.62 & 0.87 & 0.87 & 0.87 & 0.75 & 0.87 & 0.75 \\
\hline Comprensión & $0.62^{* *}$ & 1 & 0.5 & 0.75 & 0.75 & 0.62 & 0.87 & 1 & 1 & 0.75 \\
\hline Involucramiento & 1 & 0.87 & 0.75 & 1 & 1 & 1 & 1 & 1 & 1 & 1 \\
\hline Aceptación & 0.75 & 1 & 0.75 & 1 & 1 & 1 & 1 & 1 & 1 & 1 \\
\hline Por cartel & 0.78 & 0.93 & 0.62 & 0.84 & 0.90 & 0.87 & 0.93 & 0.93 & 0.96 & 0.87 \\
\hline Por contenido & \multicolumn{2}{c}{0.85} & \multicolumn{2}{c}{0.73} & 0.88 & & 0.93 & 0.91 \\
\hline
\end{tabular}

${ }^{*}$ Coeficiente $\mathrm{V}$ de Aiken ${ }^{* *}$ Se señala en negritas los criterios que no alcanzaron aprobación. 
Tabla 3. Comentarios en torno a los carteles con baja aceptación*

\begin{tabular}{|c|c|c|c|}
\hline Experto & Cartel 1 & Cartel 4 & Cartel 6 \\
\hline 1. & $\begin{array}{l}\text { "El mensaje sale cortado en un } \\
\text { recuadro" }\end{array}$ & Sin observaciones & Sin observaciones \\
\hline 2. & $\begin{array}{l}\text { "El lenguaje utilizado puede } \\
\text { ser muy complejo para los } \\
\text { cuidadores" }\end{array}$ & $\begin{array}{l}\text { "El color amarillo de fondo no } \\
\text { es muy atractivo" }\end{array}$ & $\begin{array}{l}\text { "Deberías reubicar las } \\
\text { imágenes y los letreros para } \\
\text { mayor claridad" }\end{array}$ \\
\hline 3. & Sin observaciones & Sin observaciones & $\begin{array}{l}\text { "Usa imágenes diferentes" } \\
\text { [Las imágenes presentadas } \\
\text { son similares a las de otros } \\
\text { carteles] }\end{array}$ \\
\hline 4. & $\begin{array}{l}\text { "El entendimiento de la } \\
\text { información depende del nivel } \\
\text { académico del cuidador" }\end{array}$ & $\begin{array}{l}\text { "Cambiar color de fondo y usar } \\
\text { colores vivos" }\end{array}$ & $\begin{array}{l}\text { "Agregar imágenes más } \\
\text { llamativas" }\end{array}$ \\
\hline
\end{tabular}

${ }^{*}$ No se presentan las observaciones en torno al cartel 3 debido a que existió consenso en su reelaboración por parte de los expertos y los investigadores

Tabla 4. Índice de aprobación por los jueces en la segunda versión del material didáctico

\begin{tabular}{|c|c|c|c|c|c|}
\hline Contenidos & $\begin{array}{l}\text { 1. El autocuidado: } \\
\text { una práctica } \\
\text { para toda la } \\
\text { vida }\end{array}$ & $\begin{array}{l}\text { 2. Fuentes de } \\
\text { información } \\
\text { para el } \\
\text { autocuidado }\end{array}$ & $\begin{array}{l}\text { 3. Habilidades } \\
\text { que fortalecen } \\
\text { el autocuidado }\end{array}$ & $\begin{array}{l}\text { 4. Equilibrio } \\
\text { del tiempo, } \\
\text { pasatiempos, } \\
\text { bienestar y } \\
\text { cuidado }\end{array}$ & $\begin{array}{l}\text { 5. La razón y } \\
\text { beneficios del } \\
\text { autocuidado }\end{array}$ \\
\hline
\end{tabular}

\begin{tabular}{lllllllllll} 
Criterios/ & Cartel & Cartel & Cartel & Cartel & Cartel & Cartel & Cartel & Cartel & Cartel & Cartel \\
cartel* & $\mathbf{1}$ & $\mathbf{2}$ & $\mathbf{3}$ & $\mathbf{4}$ & $\mathbf{5}$ & $\mathbf{6}$ & $\mathbf{7}$ & $\mathbf{8}$ & $\mathbf{9}$ & $\mathbf{1 0}$ \\
\hline Atracción & 0.75 & 0.87 & 1 & 0.75 & 0.87 & 0.87 & 0.87 & 0.75 & 0.87 & 0.75 \\
\hline Comprensión & $0.75^{* *}$ & 1 & 0.87 & 0.75 & 0.75 & 0.75 & 0.87 & 1 & 1 & 0.75 \\
\hline Involucramiento & 1 & 0.87 & 0.75 & 1 & 1 & 1 & 1 & 1 & 1 & 1 \\
\hline Aceptación & 0.75 & 1 & 0.75 & 1 & 1 & 1 & 1 & 1 & 1 & 1 \\
\hline Por cartel & 0.81 & 0.93 & 0.84 & 0.87 & 0.90 & 0.90 & 0.93 & 0.93 & 0.96 & 0.87 \\
\hline Por contenido & \multicolumn{2}{c}{0.87} & \multicolumn{2}{c}{0.85} & 0.90 & & 0.93 & & 0.91 \\
\hline
\end{tabular}

${ }^{*}$ Coeficiente V de Aiken **Se señala en negritas los criterios que mejoraron su aprobación en relación a la primera versión.

introductoria sobre el autocuidado. El cartel 10 no afectó la aprobación por contenido del tema cinco. Sin embargo, durante la encuesta digital no se revelaron las razones de los valores tan bajos en las casillas de observaciones, por lo que se realizaron las siguientes preguntas abiertas a cuatro de los diez cuidadores para determinar los aspectos a mejorar en dichos carteles.

Para el cartel 1:

“-¿Qué cambiaría para que el cartel le resultase más atractivo?

-El color de fondo." (Dos cuidadores no modificarían algo).

“-¿Qué recomendaciones en el cartel cambiaría para que le fuera más aceptable?

-Las frases son claras, me gustan." (Los 4 cuidadores tienen una opinión similar).

"-¿Qué contenido cambiaría para que el cartel fuera más aceptable para usted?

-De pronto un poco más explicado en los dibujos." (Tres cuidadores no cambiarían algo).

Para el cartel 10: 
“-¿Qué cambiaría del cartel para que lo asociara más con su vida diaria?

-El título que está como con reflejo confunde y las burbujas de los comentarios son confusos." (Tres cuidadores no cambiarían algo).

Debido a la naturaleza de los comentarios se consideró que, tras su modificación, el MD superó la fase de validación.

Tabla 5. Índice de aprobación por los cuidadores en la primera versión del material didáctico

\begin{tabular}{|c|c|c|c|c|c|c|c|c|c|c|}
\hline \multirow{2}{*}{$\begin{array}{l}\text { Contenidos } \\
\text { Criterios/ } \\
\text { cartel* }\end{array}$} & \multicolumn{2}{|c|}{$\begin{array}{l}\text { 1. El } \\
\text { autocuidado: } \\
\text { una práctica } \\
\text { para toda la } \\
\text { vida }\end{array}$} & \multicolumn{2}{|c|}{$\begin{array}{l}\text { 2. Fuentes de } \\
\text { información } \\
\text { para el } \\
\text { autocuidado }\end{array}$} & \multicolumn{2}{|c|}{$\begin{array}{l}\text { 3. Habilidades } \\
\text { que fortalecen } \\
\text { el autocuidado }\end{array}$} & \multicolumn{2}{|c|}{$\begin{array}{l}\text { 4. Equilibrio } \\
\text { del tiempo, } \\
\text { pasatiempos, } \\
\text { bienestar y } \\
\text { cuidado }\end{array}$} & \multicolumn{2}{|c|}{$\begin{array}{l}5 \text {. La razón y } \\
\text { beneficios del } \\
\text { autocuidado }\end{array}$} \\
\hline & $\begin{array}{c}\text { Cartel } \\
1\end{array}$ & $\begin{array}{c}\text { Cartel } \\
2\end{array}$ & $\begin{array}{c}\text { Cartel } \\
\mathbf{3}\end{array}$ & $\begin{array}{c}\text { Cartel } \\
4\end{array}$ & $\begin{array}{c}\text { Cartel } \\
5\end{array}$ & $\begin{array}{c}\text { Cartel } \\
6\end{array}$ & $\begin{array}{c}\text { Cartel } \\
7\end{array}$ & $\begin{array}{c}\text { Cartel } \\
8\end{array}$ & $\begin{array}{c}\text { Cartel } \\
9\end{array}$ & $\begin{array}{c}\text { Cartel } \\
10\end{array}$ \\
\hline Atracción & $0.60 * *$ & 0.70 & 1 & 1.0 & 0.95 & 0.80 & 0.90 & 0.80 & 1 & 0.75 \\
\hline Comprensión & 0.85 & 0.80 & 1 & 0.90 & 0.90 & 0.80 & 1 & 0.80 & 1 & 0.70 \\
\hline Involucramiento & 0.65 & 0.70 & 1 & 0.85 & 1 & 0.70 & 0.85 & 0.70 & 0.90 & 0.60 \\
\hline Aceptación & 0.55 & 0.85 & 1 & 0.90 & 1 & 0.70 & 0.90 & 0.85 & 1 & 0.7 \\
\hline Por cartel & 0.65 & 0.70 & 1 & 0.91 & 0.96 & 0.75 & 0.91 & 0.78 & 0.97 & 0.68 \\
\hline Por contenido & \multicolumn{2}{|c|}{0.67} & \multicolumn{2}{|c|}{0.95} & \multicolumn{2}{|c|}{0.85} & \multicolumn{2}{|c|}{0.84} & \multicolumn{2}{|c|}{0.82} \\
\hline
\end{tabular}

${ }^{*}$ Coeficiente $\mathrm{V}$ de Aiken ${ }^{* *}$ Se señala en negritas los criterios que no alcanzaron aprobación.

\section{DISCUSIÓN}

Se mejoró el diseño del material didáctico conforme a las recomendaciones de los expertos en el área y particularmente a los intereses de los cuidadores primarios. Estos resultados concuerdan con lo que Ziermendoff expone como el principio básico de la validación de $\mathrm{MD}$ : el público a quien está dirigido siempre tiene la razón ${ }^{27}$. Sin embargo, hubo una excepción cuando los cuidadores no propusieron una modificación severa al MD, como en el caso del cartel 1 que presentó un índice bajo de aceptación, que se atribuyó a su contenido abstracto sobre el autocuidado y que pudo ser un tema nuevo para varios cuidadores.

En general, los resultados de la presente investigación son consistentes con lo observado por diversos autores sobre la validación de materiales de apoyo para intervenciones educativas en el área de la salud. Tal es el caso de lo reportado recientemente por Herrera y Céspedes ${ }^{19}$, quienes en su proceso de validación de material educativo para pacientes con falla cardiaca sometieron a evaluación su folleto educativo con siete expertos y diez adultos con falla cardiaca, tras lo que obtuvieron un índice de aprobación de $70 \%$ con los expertos y de $60 \%$ con los adultos.

De forma similar, en Colombia, Correa ${ }^{33}$ sometió su cartilla educativa a validación técnica y por población para su intervención educativa en pacientes con implante de Stent coronario y reportó índices de aprobación muy altos en los mismos criterios que se evaluaron en nuestra investigación, 1.0 en la validez de contenido y 1.0 en tres de cuatro criterios de la validez por población. Asimismo, Holanda et al. ${ }^{21}$ construyeron una cartilla como MD para prevenir síndrome metabólico en 
adolescentes, la cual obtuvo en su mayoría índices de validez de contenido de 1.0 y un rango de respuestas positivas del público objetivo de entre $87.2 \%$ y $100 \%$.

Coincidimos con los tres autores anteriores, particularmente en el hecho de que la puntuación de los expertos fue mayor a la que reportan los cuidadores, lo que corresponde a la evaluación cuantitativa. Por otra parte, en las observaciones cualitativas es común observar modificaciones solicitadas que se centran en aspectos formales como: el uso de colores más llamativos, el tamaño de la letra y modificaciones a las imágenes, de esto dan prueba los resultados de Salazar et al. en $2012^{18}$, Correa en $2014^{33}$ y Solano et al. en $2019^{20}$, por mencionar algunos.

Limitaciones y alcance: La principal limitación observada fue la falta de publicaciones sobre la validación de material didáctico dirigido a cuidadores de personas con ECNT, así como de criterios de evaluación unificados en las publicaciones, lo que pone de manifiesto un acotado campo de interés. Cabe destacar como fortaleza de la presente investigación la disposición de materiales unificados en criterios, los cuales resultan atractivos para los cuidadores, pues se encuentran en un lenguaje aceptado por los mismos.

\section{CONCLUSIÓN}

Tras concebir el acto educativo como un procedimiento de salud surge la posibilidad y el compromiso de mejorar su desarrollo con el fin de poner a disposición de los usuarios del sistema de saludy de sus cuidadores el mejor tratamiento disponible, a costo accesible, conla mayor efectividady en cualquier entorno. Es en este punto que el diseño, validación y mejora del material didáctico para las IE encuentra su relevancia.

Se aclara que con el MD no se trata de cubrir las necesidades en salud de los cuidadores primarios reportadas en la literatura actual. Por el contrario, el MD es un recurso de apoyo en una propuesta de intervención educativa de enfermería que busca mejorar elementos en desarrollo o poco desarrollados de un grupo específico de cuidadores.

El objetivo de la presente investigación fue diseñar y validar el MD de apoyo en una propuesta de IE para mejorar el autocuidado de cuidadores de personas con ECNT. Tras ajustar elementos de la atracción y comprensión de los carteles se concluyó que el MD es adecuado para su implementación en una propuesta de IE.

\section{RESPONSABILIDADES ÉTICAS}

Protección de personas y animales. Los autores declaran no haber realizado experimentos en seres humanos ni en animales para el desarrollo de esta investigación.

Conflicto de intereses. Los autores declaran no tener conflicto de intereses.

Financiamiento. No se recibieron fondos de instituciones privadas o gubernamentales.

Agradecimientos. A los cuidadores del grupo de Familias viviendo con diabetes por su apoyo desinteresado en la presente investigación. A la Dra. Liliana González Juárez por compartir su experiencia en la validación de material didáctico y fraterno seguimiento a esta investigación. A la Lic. Saidy Ramírez Pariente por su apoyo técnico y comentarios al presente escrito.

\section{REFERENCIAS}

1. Organización Mundial de la Salud. Informe mundial sobre la diabetes. Resumen de orientación. Ginebra: OMS; 2016. http://bit.ly/2NgjnET 
2. International Labour Organization. Care work and care jobs for the future of decent work. Geneva: ILO; 2018. http://bit.ly/32fxvlZ

3. Mathers CD, Loncar D. Projections of global mortality and burden of disease from 2002 to 2030. PLoS Med. 2006; 3(11): 2011-30. https://doi.org/10.1371/journal.pmed.0030442

4. Zamarripa-Esparza EA, Tamez-Valdez BM, Ribeiro-Ferreira M. Repercusiones del cuidado informal en la vida laboral y personal de las mujeres cuidadoras. AZARBE. 2017; (6): 47-56.

https://bit.ly/zeqxjKs

5. Centeno-Soriano C. Cuidar a los que cuidan: qué y cómo hacerlo. Alcalá: Alcalá grupo editorial; 2004 .

6. Instituto Nacional de Estadística y Geografía. "Estadísticas a propósito del día de las y los cuidadores de personas dependientes (2 de marzo)". México. 2017. http://bit.ly/34yh5aa

7. Herdman TH (Ed.). NANDA Internacional. Diagnósticos enfermeros. Definiciones y clasificación. 2012-2014. Barcelona: Elsevier. 2012.

8. Salazar-Montes AM, Murcia-Paredes LM, Solano-Pérez JA. Evaluación e intervención de la sobrecarga del cuidador informal de adultos mayores dependientes: Revisión de artículos publicados entre 1997-2014. Arch. med. 2016; 16(1): 144-54. https://bit.ly/3rCBg2m

9. Baker PRA, Francis DP, Hairi NN, Othman S, Choo WY. Interventions for preventing abuse in the elderly. Cochrane Database Syst Rev. 2016; 8(CDo10321): 1-103.

https://doi.org/10.1002/14651858.CD010321.pub2

10. Maayan N, Soares-Weiser K, Lee H. Atención de relevo para los pacientes con demencia y sus cuidadores. Cochrane Database Syst Rev. 2014; 1(CDoo4396): 1-38.

https://doi.org/10.1002/14651858.CD004396.pub3

11. Acuña-Aguilarte PM, Renó-Céspedes JS, Chichay-Torres L, Álvarez-Rivero MB, Muñoz Pérez J. Fotoeducación para cuidadores de niños y adolescentes cubanos con alto riesgo de cáncer cutáneo. MediMay. 2015; 21(1): 48-56. https://bit.ly/38tqrYY

12. Da Silva-Demoro CC, Mendes-Bertoncello Fontes C, Dos Santos-Trettene A, Iwanow-Cianciarullo T, Marques-Lazarini I. Applicability of Orem: training of caregiver of infant with Robin Sequence. Rev Bras Enferm. 2018; 71(suppl 3): 1469-73. https://doi.org/10.1590/0034-7167-2016-0562

13. Vega-Hernández S, López-Mora GE, Rodríguez-Duarte SJ, Serna-Galarza M, López-Jaramillo AM, Fernández-Orozco Y. Grupo psicoeducativo en cuidados paliativos para el paciente cardiópata y su familia. Rev Mex Enf Cardiol. 2016; 24(Esp): 24-7. https://bit.ly/3eF1LRo

14. Araújo-Moreira AC, Da Silva MJ, Tirado-Darder JJ, Coutinho JFV, Osawa-Vasconcelos MI, BragaMarques M. Effectiveness of an educational intervention on knowledge-attitude-practice of older adults' caregivers. Rev. Bras. Enferm. 2018; 71(3): 1055-62.

http://dx.doi.org/10.1590/0034-7167-2017-0100

15. Mena-Aragón E, Sánchez-Sarduy M, Reyes-Quintana Y, García-Martín D. Eficacia de intervención educativa en conocimientos de cuidadores para prevenir el maltrato del adulto mayor. Rev Cuba Enf. 2016; 32(2): 1-11. https://bit.ly/38B7yDE

16. Sousa-dos Santos PD, de Souza-Araújo ZM, Barroso-Diógenes LM, Peixoto-de Caldas JM, AlvesFerreira Rodrigues K, Frota-Carneiro R. Capacitación del familiar cuidador con la aplicación de la Tecnología Educativa en Salud. Rev. Bras. Enferm. 2018; 71(3): 1135-43.

https://doi.org/10.1590/0034-7167-2017-0163

17. Rivas-Herrera JC, Ostiguín-Meléndez RM. Cuidador: ¿concepto operativo o preludio teórico? Enferm. univ. 2011; 8(1): 49-54. https://bit.ly/3lgZe2A 
18. Salazar-Coronel AA, Shamah-Levy T, Escalante-Izeta EI, Jiménz-Aguilar A. Validación de material educativo: estrategia sobre alimentación y actividad física en escuelas mexicanas. Rev Esp Comun Salud. 2012; 3(2): 96-109. https://bit.ly/3cqKV5H

19. Herrera-Guerra HP, Céspedes-Cuevas VM. Diseño y validación de material educativo dirigido a adultos con falla cardíaca. Rev. cienc. cuidad. 2020; 17(1): 31-42.

https://doi.org/10.22463/17949831.1366

20. Solano $\mathrm{MH}$, Casas C, Guarín A. Experiencia de validación de material educativo para pacientes anticoagulados en un servicio de consulta externa. Repert med cir. 2019; 28(2): 97-104.

https://doi.org/10.31260/RepertMedCir.v28.n2.2019.917

21. Holanda-De Maura I, Rodrigues-da Silva AF, Do Espírito Santo-Holanda Rocha A, De OliveiraLima LH, Magalhães-Moreira TM, Vilarouca-da Silva AR. Construcción y validación de material educativo para prevención de síndrome metabólico en adolescentes. Rev. Latino-Am. Enfermagem. 2017; 25: 1-8. https://doi.org/10.1590/1518-8345.2024.2934

22. Sánchez-Martínez RT, Molina-Cardona EM, Gómez-Ortega OR. Intervenciones de enfermería para disminuir la sobrecarga en cuidadores: un estudio piloto. Rev Cuid. 2016; 7(1): 1171-84. http://dx.doi.org/10.15649/cuidarte.v7i1.251

23. Pérez-Rodríguez M, Álvarez-Gómez T, Martínez-Badaló EJ, Valdivia-Cañizares S, Borroto-Carpio I, Pedraza NH. El síndrome del cuidador en cuidadores principales de ancianos con demencia Alzhéimer. Gac Méd Espirt. 2017; 19(1): 1-12. https://bit.ly/2OMBTYG

24. Velazquez-Moreno E, González-Velázquez MS, De la Peña-León B, Soria-Flores A. Calidad de las intervenciones educativas dirigidas al cuidador primario. Una revisión integradora. Rev Enferm Inst Mex Seguro Soc. 2019; 27(4): 223-9. https://bit.ly/3qCfMkR

25. Toledo-Vila HJ, Borroto-Chao R, Libca-Flores M, Núñez-Tabares C. Determinación de prioridades en salud con participación social. Rev Cubana Med Gen Integr. 1996; 12(3): 222-8.

https://bit.ly/3oCjC2V

26. Guerra-García M, Alva M./ Fondo de las Naciones Unidas para la Infancia. Guía metodológica y video de validación de materiales IEC. Lima, Perú: UNICEF; 2003. https://uni.cf/3vhXZmy

27. Ziemendorff S, Krause A. Guía de validación de materiales educativos (con enfoque en materiales de educación sanitaria). Chiclayo, Perú: Programa de Agua y Saneamiento PROAGUA/GTZ; 2003. https://doi.org/10.13140/RG.2.1.3560.1129

28. Organización Panamericana de la Salud. Guía para el diseño, utilización y evaluación de material educativo en salud. Washington, E.U.A.: OPS; 1984. https://bit.ly/2OgNaRj

29. Cavanagh SJ. Modelo de Orem: aplicación práctica. España: Ciencias y Técnicas. 1993.

30. Orem D. Self-care and health promotion: understanding self-care. En: McLaughlin-Renpenning K, Taylor SG (Eds.). Self-care theory in nursinq: selected papers of Dorothea Orem. New York: Springer Publishing Company, Inc.; 2003.

31. Pender NJ. Health promotion in nursing practice. Stamford, Conn, EE. UU.: Applenton \& Lange; 1996.

32. Martínez-González O, Palmar Santos AM, Pedraz- Marcos A. La metodología de un proyecto de educación para la salud. En: Palmar-Santos AM. Métodos educativos en Salud. España: Elsevier. 2014.

33. Correa-Tello KJ. Diseño y validación de material para una intervención educativa en pacientes con implante de Stent coronario. Perspectivas Educativas. 2014; 7(1): 159-70.

34. Secretaria de Salud. Reglamento de la Ley General de Salud en Materia de Investigación para la Salud. México: SSA; Última modificación 2014.

35. Beauchamp TL, Childress JF. Principios de Ética Biomédica. $4^{\text {a }}$ ed. Barcelona: Masson; 1994. 